\title{
Monte Carlo Studies on Proton Computed Tomography using a Silicon Strip Detector Telescope
}

\author{
L. R. Johnson*, B. Keeney*, G. Ross*, H. F.-W. Sadrozinski* (Senior member, IEEE), \\ A. Seiden", D. C. Williams*, L. Zhang* \\ V. Bashkirov ${ }^{\dagger}$, R. W. Schulte ${ }^{\dagger}, K_{\text {S Shahnazi }}^{\dagger}$ \\ *Santa Cruz Institute for Particle Physics, UC Santa Cruz, CA 95064 \\ ${ }^{\dagger}$ Loma Linda University Medical Center, Loma Linda, CA 92354
}

\begin{abstract}
Monte Carlo computer programs such as the GEANT4 toolkit include precise models of particle interactions and can be an invaluable tool for studying the feasibility of new imaging techniques such as proton computed tomography (pCT). Presented in this paper is a comparison of laboratory data with GEANT4 predictions for the imaging characteristics of a simple metal object using a particle beam and a silicon particle detector.
\end{abstract}

\section{INTRODUCTION}

Computed tomography (CT) has become an important tool in medical imaging. Reconstruction methods allow doctors to make detailed pictures of everything from the brain to the heart. $\mathrm{CT}$ is especially important in imaging irregular growths or damaged and diseased tissue for treatment purposes. However, traditional CT scans using X-rays as probes have a disadvantage of a relatively high radiation dosage. A possible alternative is proton computed tomography ( $\mathrm{pCT}$ ), an imaging technique that substitutes protons for X-rays. Imaging with protons could have the advantage of providing similar quality reconstruction with much less dose. [13]

In order to study the feasibility of pCT or other alternative imaging techniques, it is important to be able to understand the proton transmission images seen in the lab. An accurate analysis of laboratory data requires the use of detailed computer simulations. GEANT4 is an excellent tool for this purpose [7]. In this paper we will examine the correlation between laboratory data and GEANT4 simulations.

\section{EXPERIMENT}

\section{A. Setup}

Data for our initial experiments was taken using the medical proton synchrotron at Loma Linda University Medical Center. A monochromatic beam of $250 \mathrm{MeV}$ protons is degraded by a $25.4 \mathrm{~cm}$ thick wax block, resulting in protons with mean energy of approximately $130 \mathrm{MeV}$. The protons then pass through a $5.0 \mathrm{~cm}$ long aluminum tube (outer diameter $\mathrm{OD}=3.0 \mathrm{~cm}$, inner diameter ID $=0.68 \mathrm{~cm}$ ) resting on a polystyrene holder and placed $25 \mathrm{~cm}$ behind the wax block. The protons were then detected by two sets of silicon strip detectors (SSD), one placed directly behind the tube, and the second $27.3 \mathrm{~cm}$ from the Prst as shown in Figure 1.
The wax material of the beam degrader has a physical density of $0.926 \mathrm{~g} / \mathrm{cm}^{3}$. The water-equivalent thickness in terms of

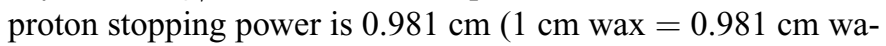
ter) for high energy protons. The elemental composition of the wax was described in [12], and is very close to that of polyethylene, which is, by weight, $0.143711 \mathrm{H}$ and $0.856289 \mathrm{C}$.

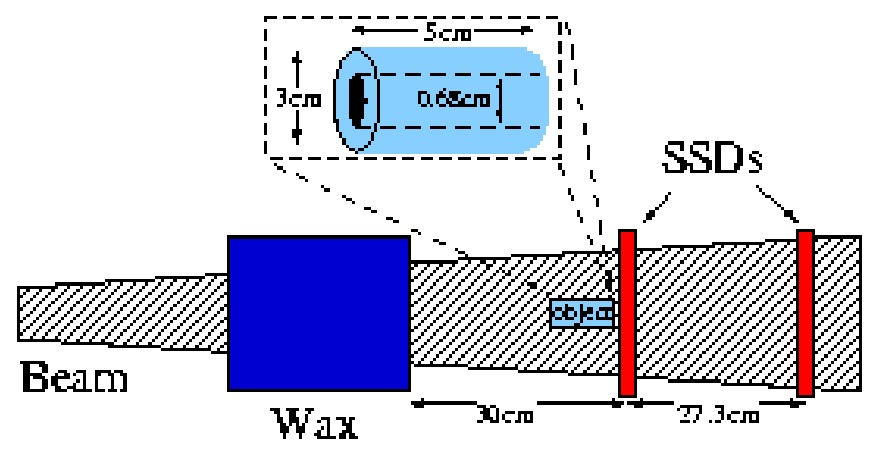

Fig. 1. Experimental setup used for the study of Proton Computed Tomography (pCT).

Each SSD consists of a pair of single-sided silicon strip detectors, originally developed for the gamma-ray large area space telescope (GLAST). [3] Each single-sided detector is composed of 320 thin strips of silicon aligned parallel to each other in a plane. In each detector plane, two of these single sided detectors are arranged such that they have orthogonal strip orientation. The detectors are $400 \mu \mathrm{m}$ thick, with a pitch of 194 microns, and outer dimensions of $6.4 \mathrm{~cm}$ by $6.4 \mathrm{~cm}$. These detectors measure the trajectory of the protons (incident $\mathrm{x}$ and $\mathrm{y}$ position and direction) as well as their energy. The Prst is determined from strip-hit information, and the latter by measuring the charge deposited in the detector. [11]

\section{B. Energy Measurement}

For each event there are three kinds of quantities recorded: event number, strip hit information for each detector, and time over threshold (TOT). The strip hit information tells which strips in the SSD are traversed by protons. Since each detector plane contains two silicon detectors with strips oriented in perpendicular directions, both $\mathrm{x}$ and $\mathrm{y}$ trajectories can be measured with high precision. 
As protons pass through the detector, they deposit some amount of charge in the silicon that depends on the energy of the proton. This energy creates a signal which is transmitted to a binary chip with a threshold that can be set for every channel, and has a fast output of TOT. The TOT depends linearly on the input charge up to about $100 \mathrm{fC}$. By using specipc proton energies and measuring the corresponding TOT, an experimental calibration curve was obtained. This curve, shown in Figure 2 , was used to calculate the proton energies from TOT in our experiment. [11]

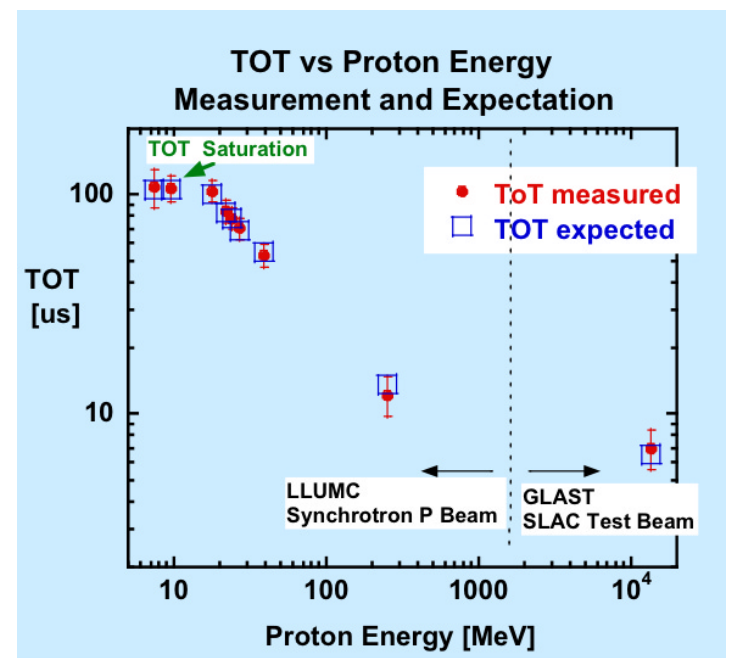

Fig. 2. By using specibc proton energies and measuring the corresponding time over threshold (TOT), this experimental calibration curve was obtained. It was used to calculate the proton energies from TOT in our experiment.

In the process of recording the data, certain events were excluded from analysis. One feature of the integrated circuit used for the readout ${ }^{1}$ is that it only returns one TOT value per detector regardless of how many strips are hit. Multiple-hit events are generally from individual protons passing through an area at the edge of two strips in a plane, in which case the deposited charge is shared between strips. Therefore, in order to get good energy resolution, we exclude any events that activated more than one strip per plane. The resulting average energy vs position measurements are shown in Figure 3.

\section{Simulation}

The Monte Carlo simulation program GEANT4 was used to model our simple experimental setup. GEANT4 was developed by RD44 with the goal of providing a toolkit to simulate the passage of particles through matter. Although GEANT4 was primarily intended (and tested) for the simulation of highenergy physics experiments, it can be applied to a wide variety of applications, including medical physics. For this reason, it is

\footnotetext{
${ }^{1}$ The circuit used here was designed for GLAST [10]. It is a low-power design meant for use in a satellite. An ampliper custom designed for the pCT application is currently in development as a replacement.
}

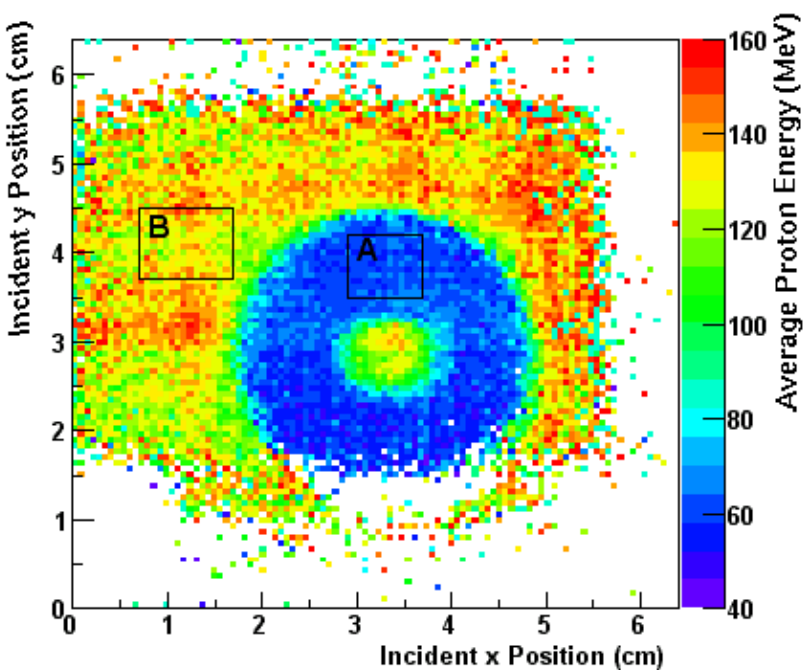

Fig. 3. Experimentally observed proton energy, converted from the TOT values, and including multiple-hit cuts and chip calibration.

important to verify its accuracy at the types of energies useful for pCT. [1]

The parameters for the simulation were matched to the physical setup described in Section II-A. The simulated beam consisted of $250 \mathrm{MeV}$ monochromatic protons. The dimensions for the wax block, aluminum tube and silicon detectors were identical to the experimental setup, as were the alignment and spacing of these objects. The wax was modeled as a simple solid made of $85.6 \%$ carbon, $14.4 \%$ hydrogen, with density $\rho=0.926 \mathrm{~g} / \mathrm{cm}^{3}$. Each detector was modeled as two thin sheets of silicon adjacent to each other. At the plane of the detector, the energy, $\mathrm{x}$ and $\mathrm{y}$ positions and incident angle were recorded for each proton. The data was then analyzed in a manner similar to the experimental data.

\section{COMparison of Experiment With Monte CARlo}

\section{A. Multiple Scattering}

As particles travel through matter, they interact primarily with nuclei via the Coulomb interaction, and scatter. If the material is thick enough, many small deßections combine to produce scattering angles in a Gaussian distribution of predictable width:

$$
\theta_{0} \propto \frac{z \sqrt{x / X_{0}}}{\beta c p}
$$

Here, $p$ is the momentum, $\beta c$ the velocity, $z$ the charge number of the incident particle and $x / X_{0}$ is the thickness of the medium in radiation lengths. [4] We would expect to see this Gaussian behavior in both the data and the simulation.

For this measurement, we relaxed the requirement of only one hit per plane discussed in Section II-B. This is to avoid 


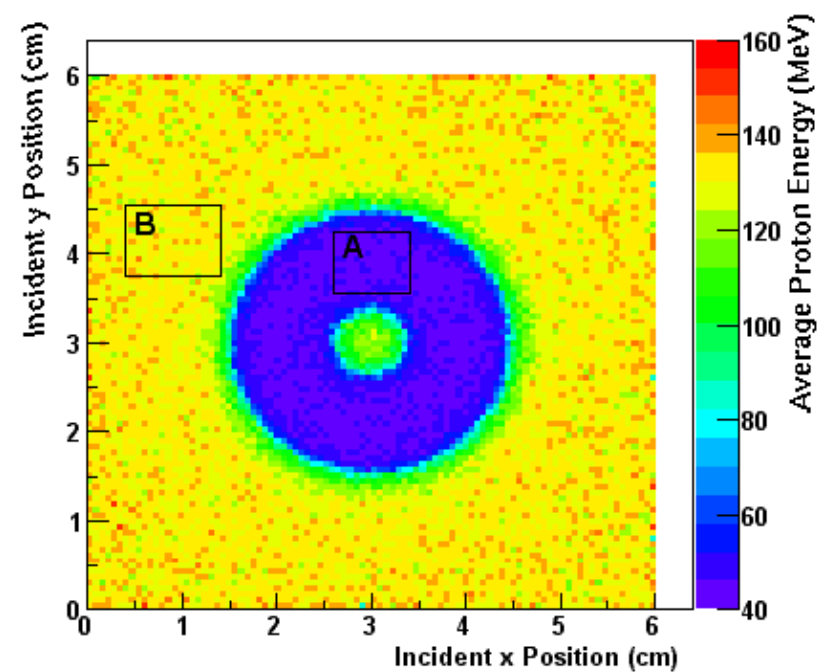

Fig. 4. Simulated proton energy vs. position without cuts.

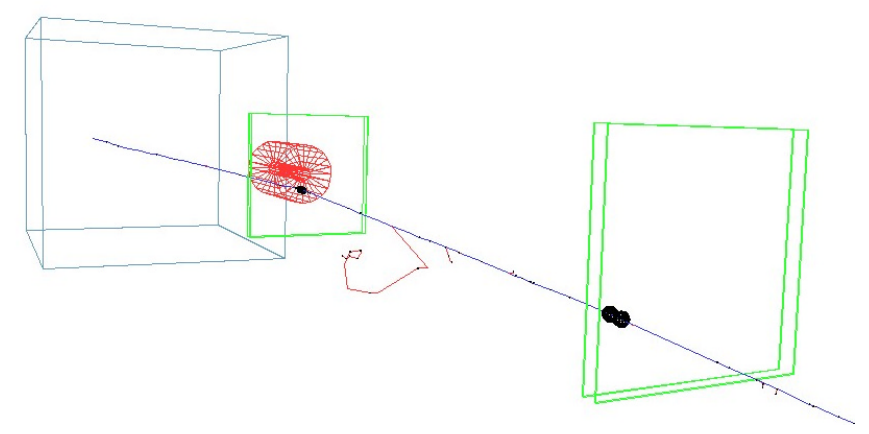

Fig. 5. T-D image of one simulated proton being scattered by the wax and aluminum tube, generated using GEANT4. The wax is the large box, the single sided SSDs are shown in green, the aluminum tube in red. The points where the proton impacted the detectors are shown in black. The red lines are secondary particles created by GEANT4 (delta rays).

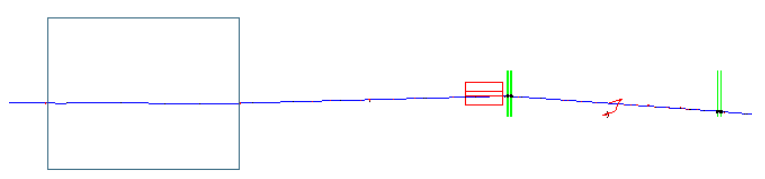

Fig. 6. Two-D side view of event in Figure 5. It is easy to see the angle of deßection between the incident proton and the bnal path after it has passed through the aluminum tube.

bias, since particles traveling at an angle are more likely to hit multiple strips. In order to extract information about the multiple scattering of the protons in the experimental setup, it was important to be able to match events in the brst and second detector planes. By comparing the $\mathrm{x}$ and $\mathrm{y}$ position of the proton hit in the brst plane to the position in the second plane, the incident angle from the norm was extracted and recorded.
In running the simulation, one of the parameters recorded for each proton was the incident angle at the plane of the brst detector. However, in order to compare the simulation with experiment it is important to make the same set of cuts on the both sets of data. Therefore we required that the Ógoodóvents in the simulation must also impact both detector planes. This then excluded any protons that scatter with an angle large enough to miss the second detector.

We compared scattering data for protons that passed through the aluminum tube (region A in Figures 3 and 4) separately from scattering data for protons that did not pass through the aluminum (region B in Figures 3 and 4). The comparisons for each region are shown in Figure 7.

There is good agreement between the experimental and simulated results. Both distributions are roughly Gaussian in shape, and centered at zero. The spread in scattering angles in region A is signipcantly wider than the spread in region B. This is expected, since the scattering angle depends on the amount and density of material traversed. GEANT4 appears to accurately simulate multiple scattering for protons at these energies.

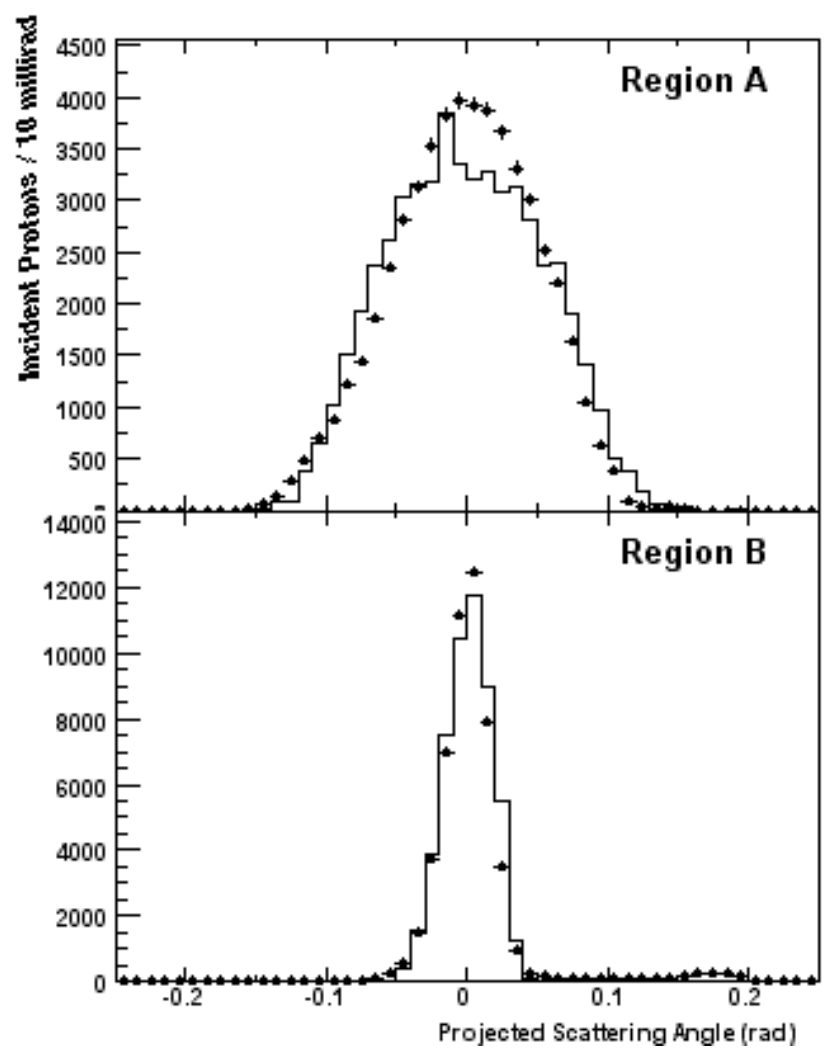

Fig. 7. Comparison of the scattering angle of exiting protons for experiment (points) and simulation (histogram) in regions $\mathrm{A}$ and $\mathrm{B}$ as indicated in Figures 3 and 4 . The area under the histograms was normalized to correspond to the area of the experimental curves. 


\section{B. Energy Loss}

Protons lose energy in matter mostly through atomic excitation and ionization. This process is well described by the BetheBloch equation. At the energies relevant to $\mathrm{pCT}$, this equation gives the stopping power (i.e. mean rate of energy loss) to be approximately,

$$
-\frac{d E}{d x} \propto z^{2} \frac{Z}{A} \rho
$$

where $Z$ and $A$ are the atomic number and atomic mass of the absorber, $z$ the charge of the incident particle and $\rho$ the specipc gravity. [4] Using the information about the wax provided in Sections II-A, the theoretical change in energy of the 250 $\mathrm{MeV}$ protons can be calculated. We expect the energy of the protons that have passed through the wax only (region B in Figures 3 and 4) to be approximately $136 \mathrm{MeV}$ while those passing through both the wax and the aluminum (region A in Figures 3 and 4) to be about $57 \mathrm{MeV}$.

For the experimental energy measurement, the set of cuts outlined in Section II-B were used. For the remaining ÓgoodÓ events, the proton energies at the Prst detector plane were calculated from the TOT using the calibration curve as explained in Section II-B.

The energy of the simulated protons was recorded at the location of the Prst detector plane. We again excluded events that did not impact both detector planes, so as to be consistent with the experimental cuts.

As in section IV-A, we considered the data from the protons passing through region $\mathrm{A}$ in Figures 3 and 4 separately from those passing through region B. The results are shown in Figure 8.

It is obvious from Figure 8 that the simulation and experimental energy data do not agree as well as the multiple scattering sets. In region B there is a heavy left tail to the experimental data that the simulation does not predict. The existence of this tail seems unusual, as theoretically there should not be this many low energy protons if these protons passed through the wax only. These tail events could be from several sources. First, some of these could be protons that passed through the aluminum and scattered at larger angles before depositing energy in this region. However, since the number of protons scattering at large angles should be small, this is likely only a small portion of these events. It is also possible that these are events in which signibcant charge sharing occurred, but did not register as having readings in more than one strip. Another possibility is that these events originated from the pile-up of two protons that were registered as a single proton.

There is also a discrepancy in the average energy of experiment and simulation in each region. In region A, the experimental results seem too high, while the simulated results much too low. In region B, though the peaks are closer, the simulated distribution is much more asymmetrical then the experimental. These errors could come from a number of sources. It is possible that the wax and the aluminum are not properly treated in the simulation, perhaps because their composition and density are not correctly speciped. It is difbcult, however, to explain the size of the discrepancy in energy loss due to these sources alone. This could indicate errors in the TOT to energy calibration curve. We used an extrapolation of the curve to Pt the data. However, there are no calibration points in the range of energies that we are seeing in our experiment, approximately $40-160 \mathrm{MeV}$, (see Figure 2) so we may not be using the correct calibration at these energies. In addition, the beam may have been contaminated with low-energy protons.

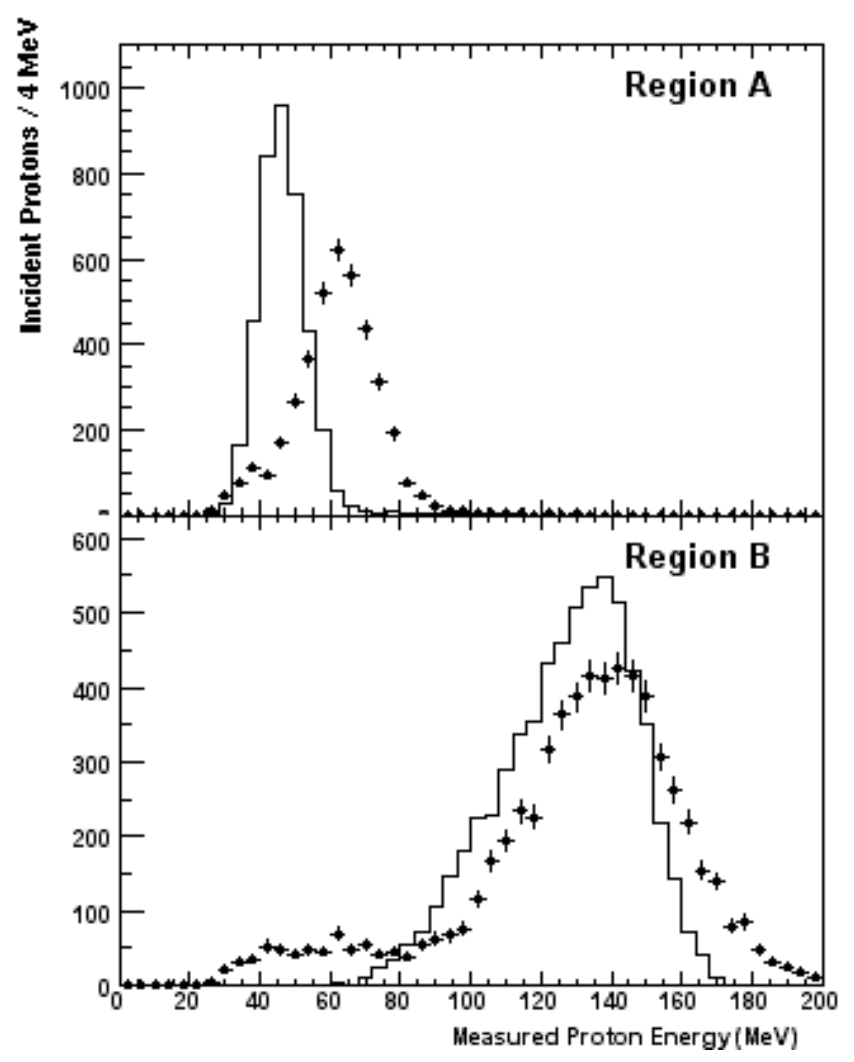

Fig. 8. Comparison of the energy of exiting protons for experiment (points) and simulation (histogram) in regions $A$ and $B$ as indicated in Figures 3 and 4. The area under the histograms were normalized to correspond to the area of the experimental curves.

\section{FUTURE WORK}

In continuing this work, it is Prst important to understand the discrepancies between the GEANT4 simulation and experiment. The TOT calibration needs to be evaluated and improved, and calibration in the detectors needs to be performed on a channel by channel basis. The wax and aluminum materials also needs to be examined to determine if we are modeling them properly. We may also need to adjust the modeling of the beam. Once we understand these factors, we can move on to modeling more complicated experimental setups. First, we will look at 
an object imbedded in wax and compare this to a corresponding experimental setup. Eventually, we would like to simulate a more complicated phantom, with smaller embedded objects and perhaps with very small density ßuctuations like those that typically appear in human soft tissues.

More accurate modeling of the detectors must also be considered in future simulations. In particular, the addition of individual strips of silicon arranged as in the real detector, instead of single planes of silicon, is important so that the simulation more closely resembles the experimental setup. This would also enable us to examine issues such as charge sharing between strips. Additionally, including new types of detectors with better energy resolution will be vital in discovering how to get the best resolution with the lowest dose.

\section{REFERENCES}

[1] Geant4 results and publications, wwwinfo.cern.ch/asd/geant4/geant4.html, 2002.

[2] A. M. Cormack and Koehler. Phys. Med Biol., 21/4:560Đ569,1976.

[3] E. do Couto e Silva, G. Godfrey, P. Anthony, R. Arnold, H. Arrighi, and E. Bloom et al. Results from the beam test of the engineering model of the GLAST LAT. Nucl. Instrum. Meth., A474/1:19Đ37,2001.

[4] K. Hagiwara et al. Review of Particle Physics. Physical Review D, 66:010001+, 2002

[5] K. M. Hanson et al. Phys. Med Biol., 26:965Đ983,1982.

[6] U. Scheider et al. Phys. Med Biol., 41:111Đ124,1996.

[7] S. Agostinelli et all. Geant4: A simulation toolkit. Submitted to Nucl. Inst. Meth., SLAC-PUB-9350.

[8] M. Goitein. Nucl. Instrum. Meth., 101:509Đ518,1972.

[9] Gabor T. Herman. Image Reconstruction from Projections: The Fundamentals of Computerized Tomogrophy. Academic Press, New York, NY, 1980.

[10] R. P. Johnson, P. Poplevin, H. F.-W. Sadrozinski, and E. Spencer. An Ampliber -Discriminator chip for the GLAST silicon-strip Tracker. IEEE Trans. Nucl. Sci, 45:927Đ937,1998.

[11] B. Keeney, V. Bashkirov, R. P. Johnson, W. Kroeger, H. Ohyama, H. F.-W. Sadozinski, R. W. M. Schulte, A. Seiden, and P. Spradlin. A silicon telescope for applications in nanodosimetry. IEEE Transactions on Nuclear Science, 49(4):1724Đ1727,2002.

[12] D. A. Low and K. R. Hogstrom. Phys. Med. Biol., 39:1063Đ1068,1994.

[13] Hartmut Sadrozinski. Proton Computed Tomography, IEEE MIC 2002 Invited Talk. 